\title{
Magnetism in Dopant-Free ZnO Nanoplates
}

\author{
Jung-Il Hong, ${ }^{* \dagger}$ Jiil Choi, ${ }^{\dagger}$ Seung Soon Jang, ${ }^{* \dagger}{ }^{\dagger}$ Jiyeong Gu, ${ }^{\ddagger}$ Yangling Chang, ${ }^{\dagger}$ Gregory Wortman, ${ }^{\S}$ \\ Robert L. Snyder, ${ }^{\dagger}$ and Zhong Lin Wang ${ }^{*},^{\dagger}$
}

${ }^{\dagger}$ School of Materials Science and Engineering, Georgia Institute of Technology, Atlanta, Georgia, United States

${ }^{\star}$ Department of Physics and Astronomy, California State University, Long Beach, Long Beach, California, United States

${ }^{\S}$ PANalytical, 117 Flanders Road, Westborough, Massachussets, United States

\section{Supporting Information}
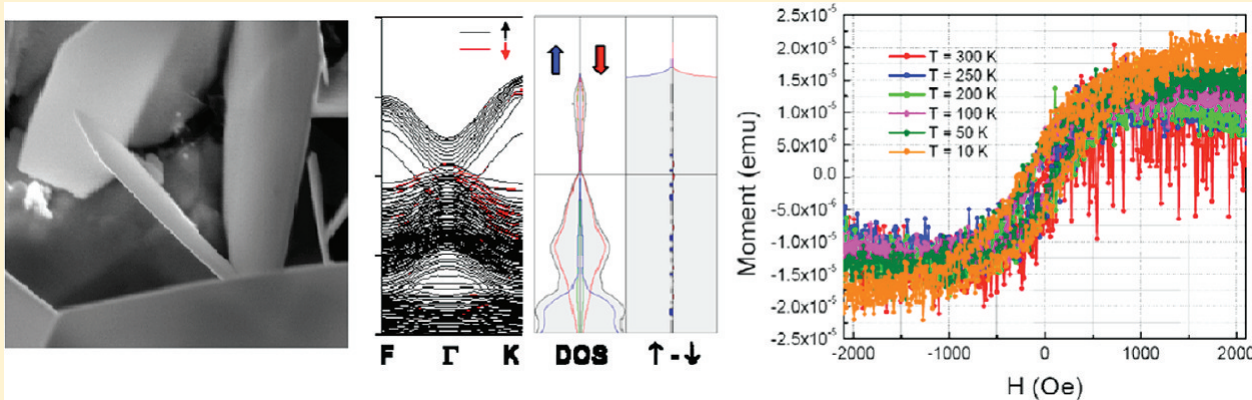

ABSTRACT: It is known that bulk $\mathrm{ZnO}$ is a nonmagnetic material. However, the electronic band structure of $\mathrm{ZnO}$ is severely distorted when the $\mathrm{ZnO}$ is in the shape of a very thin plate with its dimension along the $c$-axis reduced to a few nanometers while keeping the bulk scale sizes in the other two dimensions. We found that the chemically synthesized $\mathrm{ZnO}$ nanoplates exhibit magnetism even at room temperature. First-principles calculations show a growing asymmetry in the spin distribution within the distorted bands formed from $\mathrm{Zn}(3 \mathrm{~d})$ and $\mathrm{O}(2 \mathrm{p})$ orbitals with the reduction of thickness of the $\mathrm{ZnO}$ nanoplates, which is suggested to be responsible for the observed magnetism. In contrast, reducing the dimension along the $a$ - or $b$-axes of a $\mathrm{ZnO}$ crystal does not yield any magnetism for $\mathrm{ZnO}$ nanowires that grow along $c$-axis, suggesting that the internal electric field produced by the large $\{0001\}$ polar surfaces of the nanoplates may be responsible for the distorted electronic band structures of thin $\mathrm{ZnO}$ nanoplates.

KEYWORDS: Nanoplates, $\mathrm{ZnO}$, magnetism, polar surfaces, electronic band

I nterest in the two-dimensional (2D) structure of graphene has rapidly increased in recent years due to the development of its unexpected band structure and the corresponding new physical properties. ${ }^{1,2}$ From the seemingly simple shape/ geometry change from $1 \mathrm{D}$ carbon nanotubes to $2 \mathrm{D}$ graphene, unexpected properties of the carbon structure have been discovered. ${ }^{3}$ On the other hand, while significant effort in inorganic nanomaterial research has been directed toward nanowires, that is, 1D nanostructures, ${ }^{4,5}$ we have not seen a comparable growth in activity directed toward 2D structures. One of the reasons for this is the difficulty in preparing freestanding $2 \mathrm{D}$ structures with appreciable lateral width to thickness ratio (aspect ratio). While it has been relatively easy to prepare graphene due to the weak $\pi-\pi$ interaction between the layers, the formation of free-standing layered crystalline structures of inorganic material is much more challenging due to the strong ionic bonding between the atomic layers. One way of producing a $2 \mathrm{D}$ structure with an extremely high aspect ratio would be to look at materials with highly anisotropic crystal structures such as wurtzite. The wurtzite structure of $\mathrm{ZnO}$ has hexagonal symmetry and is highly anisotropic with very different surface energies between its crystal planes, hence
$\mathrm{ZnO}$ crystals usually grow in highly anisotropic shapes such as ID nanowires along the $c$-axis, ${ }^{4,6}$ nanobelts, ${ }^{7}$ or highly textured thin films. ${ }^{8}$ A few studies have reported platelet-shaped nanoparticles of $\mathrm{ZnO}$, but the aspect ratios were not high enough $(<100)$ with substantial thicknesses, ${ }^{9-12}$ or the crystalline order was not complete enough over the entire plate to be addressed as a crystalline $2 \mathrm{D}$ nanostructure. ${ }^{13,14}$

In this Letter, we report a surprising observation of magnetism in $\mathrm{ZnO}$ nanoplates with very high aspect ratios of a few hundreds to a thousand. $\mathrm{ZnO}$ is traditionally known as a nonmagnetic material. Through first-principle calculations, we show that the presence of magnetism in $\mathrm{ZnO}$ nanoplates is a size and structure induced change in the spin distribution within the distorted bands structure. This is a dramatic example of new physical properties being induced from nanoscale anisotropic shape and structure.

$\mathrm{ZnO}$ nanoplates with thicknesses at the nanometer scale were synthesized using $\mathrm{GaN}$ thin film as substrate. The

Received: August 31, 2011

Revised: November 12, 2011 
chemical growth technique developed previously for the synthesis of $\mathrm{ZnO}$ nanowires ${ }^{15}$ was employed. The substrate was floated on the surface of a $0.5 \mathrm{mM}$ solution of zinc nitrate and hexamethylenetetramine (HMTA) with the growth surface facing down, and the solution was kept at approximately $85^{\circ} \mathrm{C}$

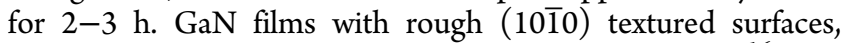
grown on $\mathrm{Si}$ wafers by pulsed laser deposition technique, ${ }^{16}$ were used as the substrate. After the reaction for $2-3 \mathrm{~h}$, the substrate was gently washed with distilled water and dried in air. $\mathrm{ZnO}$ grew in the shape of thin hexagonal platelets with their $c$-axes perpendicular to the surface of the platelets as shown in the scanning electron microscopy (SEM) images in Figure 1.
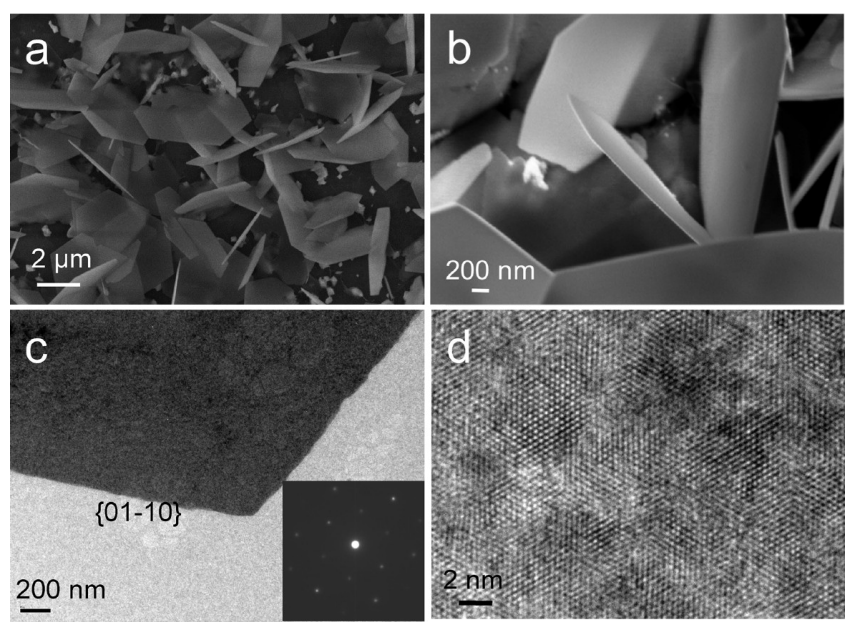

Figure 1. $\mathrm{ZnO}$ nanoplates synthesized in hexagonal plate shape by hydrothermal reactions on a GaN film. (a) Low-magnification, (b) high-magnification SEM images, (c) TEM image with electron diffraction pattern, and (d) high-resolution lattice image recorded with the electron beam normal to the nanoplate.
The thickness of the nanoplates is roughly estimated to be less than $30 \mathrm{~nm}$ at most. However, it is expected that many plates would be much thinner because the SEM tends to overestimate the thickness due to its typical resolution of a few nanometers. The average thickness is calculated to be $\sim 20 \mathrm{~nm}$ from the width of (0002) X-ray diffraction peak. The lateral widths of the nanoplates range from a few micrometers to over $10 \mu \mathrm{m}$ with aspect ratios (width/thickness) from a few hundreds to a thousand. From transmission electron microscopy (TEM) observations (Figure 1c), hexagonal shape is consistent with the symmetry of $\mathrm{ZnO}$ crystalline structure phase and is further confirmed by electron diffraction (inset of Figure 1c) as well as high-resolution TEM imaging in Figure 1d. The crystallographic orientation of the nanoplate could readily be recognized.

We note that the growth conditions of the nanoplates are the same as those for nanowire growth except for the substrates being used. When $\mathrm{ZnO}$ films were used for the substrates, $\mathrm{ZnO}$ nanowires along the $c$-axis grew in the solution. ${ }^{15}$ On the $(10 \overline{1} 0)$ surface of $\mathrm{GaN}$, the growth reaction resulted in freestanding $\mathrm{ZnO}$ nanoplates. It is thought that the epitaxial stress exerted by the $\mathrm{GaN}$ during the early growth stage of $\mathrm{ZnO}$ suppresses the growth of $\mathrm{ZnO}$ along the $c$-axis direction. When a very thin $\mathrm{GaN}$ film (with thickness less than a few nanometers) was used as the substrate, the epitaxial stress at the interface between $\mathrm{GaN}$ and $\mathrm{ZnO}$ was reduced since the $\mathrm{GaN}$ could relax to accommodate the epitaxy with $\mathrm{ZnO}$. Consequently, thicker $\mathrm{ZnO}$ nanoplates could be grown.

The electronic band structure of the $\mathrm{ZnO}$ nanoplates was calculated for various thicknesses using the density functional $(\mathrm{DFT})^{17}$ theory with general gradient approximation and the Hubbard potential $(\mathrm{GGA}+\mathrm{U})$ method. ${ }^{18}$ In order to investigate the magnetic property, the plane-wave based DFT was performed with the spin-polarized GGA+U method, ${ }^{18}$ which ensures appropriate electron correlations hybridization.
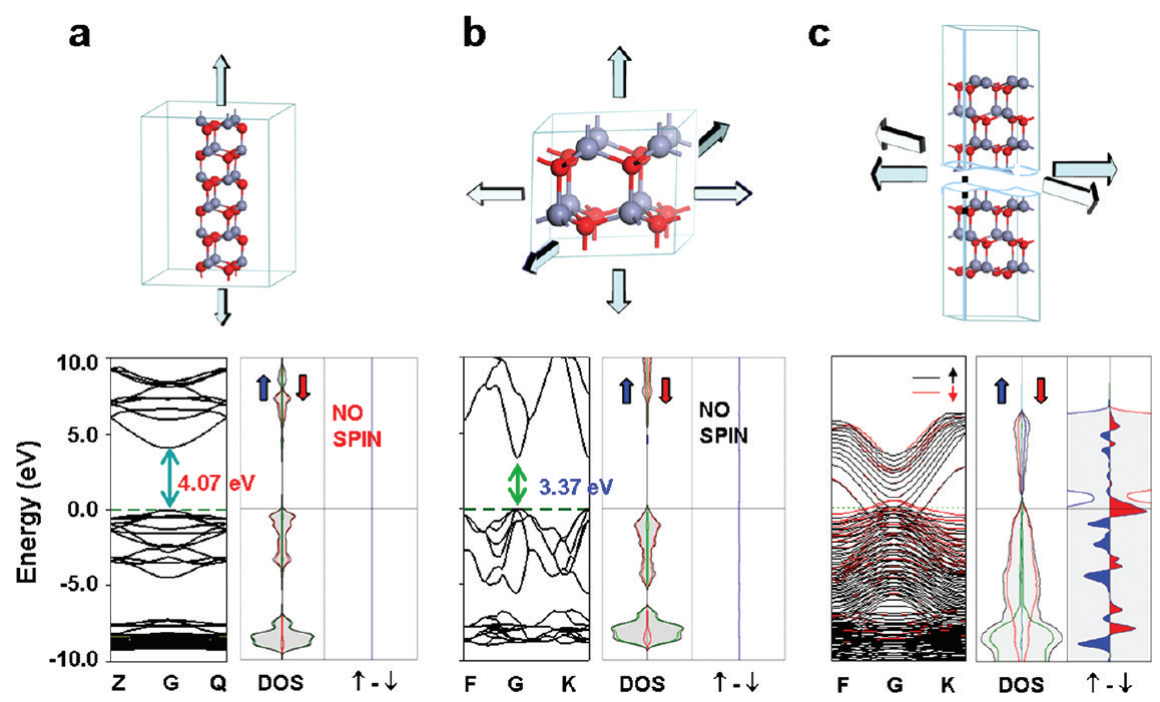

Figure 2. Calculated band diagrams and spin density of states for (a) $\mathrm{ZnO}$ nanowires, (b) bulk, and (c) nanoplates. The plane-wave based DFT with spin polarized GGA plus Hubbard parameter $\mathrm{U}$ for $\mathrm{Zn}(3 \mathrm{~d}) 12.0 \mathrm{eV}$ and $\mathrm{O}(2 \mathrm{p}) 7.0 \mathrm{eV}$ is used to ensure appropriate electron correlations hybridization. The $9 \times 9 \times 1 k$-point mesh is used for the Brillouin zone sampling. Periodic boundary conditions are applied along the directions indicated with the arrows. Red and black curves in the band diagrams represent the bands for spin up and spin down, respectively. For the $\mathrm{ZnO}$ bulk phase (b), the calculated band structure and band gap agree well with the experimental observations $(\sim 3.4 \mathrm{eV})$. The band gap becomes larger for the thin nanowire (a) but disappears for the nanoplates (c). The balance between spin up and spin down density of states breaks for nanoplates resulting in net magnetic moments. 


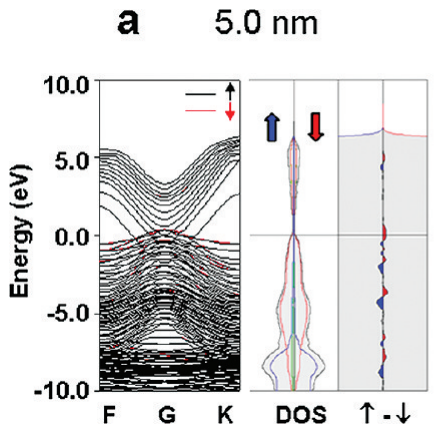

b $\quad 6.6 \mathrm{~nm}$

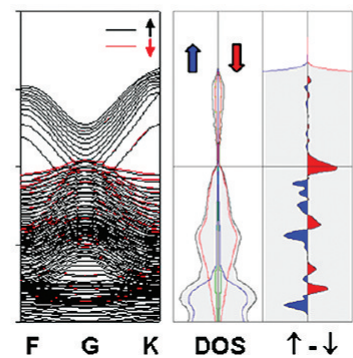

C

$8.2 \mathrm{~nm}$

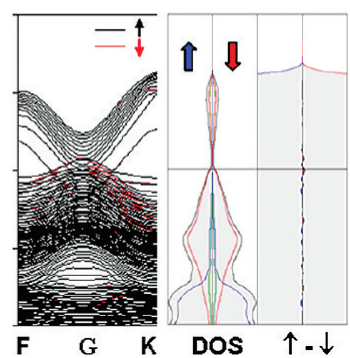

Figure 3. Calculated band structure, density of states, and spin density of states (difference between spin-up and spin-down electron density of state, $\uparrow-\downarrow)$ for the nanoplates with thicknesses of (a) 5.0, (b) 6.6, and (c) $8.2 \mathrm{~nm}$. In the DOS plots, red and green lines represent the contributions from O (2p) and $\mathrm{Zn}$ (3d) orbitals in the same scale, respectively. (b) shows greater spin density of states compared to others. For the extremely thin nanoplates with thicknesses less than $5 \mathrm{~nm}$, there is a driving force to transform the wurtzite crystal structure to the graphitic structure which carries no dipoles in the $\mathrm{ZnO}$ layers. The internal electric field reduces with the thickness of the plate, hence the reduced magnetic moment. As the thickness increases, the magnitude of the internal field would also decrease and its effect on the orbitals reduce accordingly.

Nanoplates are configured with a Zn-terminated (0001) surface and an O-terminated $(000 \overline{1})$ surface at various thicknesses. The surface reconstruction was achieved through geometry optimization in which atomic positions are relaxed via an energy minimization. Since the atomic structure in a nanoplate would be different from that in the bulk phase crystal structure due to the nearness of the surfaces on both sides, the atomic positions in a nanoplate adjusted through geometry optimization can be different from those in the bulk phase. For comparison, the band structure of the $\mathrm{ZnO}$ bulk phase and a $\mathrm{ZnO}$ nanowire were also calculated using the same method and parameters. Figure 2 shows three models of different $\mathrm{ZnO}$ geometries and the corresponding computational results. For the $\mathrm{ZnO}$ bulk phase, the calculated band structure and band gap agreed well with the known theoretical and experimental observations (Figure 2b). Alternatively, the band structure of the $\mathrm{ZnO}$ nanowire is consistent with the experimental observation of the blue shift of the photoluminescence peak for nanowires; ${ }^{19}$ its band gap $(4.07 \mathrm{eV})$ is greater than that of $\mathrm{ZnO}$ bulk phase (Figure 2a) and the band gap approches the value for bulk $\mathrm{ZnO}(3.37 \mathrm{eV})$ with increasing diameter of the nanowire.

On the contrary, in nanoplates it is found that the conduction and valence bands overlap at the Fermi level and the band gap is suppressed. Severe modifications of the band structure in core levels as well as in the levels near the Fermi surface result in unpaired spin density of states. This means that the difference in the density of states between up spin and down spin in the $\mathrm{ZnO}$ nanoplate does not vanish, leading to the development of the magnetic moment. Nanowire and bulk $\mathrm{ZnO}$ do not show any distinguishable difference in the band structures for up and down spin states, meaning that they remain nonmagnetic. The development of electronic states as well as the magnetic moment as a function of nanoplate thickness is shown in Figure 3, and the expected magnetic moments corresponding to the three thicknesses were listed in Table 1. The difference between the up spin and down spin states disappears with increasing thickness beyond $\sim 8 \mathrm{~nm}$. It is also noted that $\mathrm{O}(2 \mathrm{p})$ and $\mathrm{Zn}(3 \mathrm{~d})$ orbitals give dominant contributions to the (spin) density of states. Therefore, it is thought that the unusual hybridizations of $\mathrm{Zn}(3 \mathrm{~d})$ and $\mathrm{O}(2 \mathrm{p})$ orbitals occur in the nanoplate to form "distorted" valence bands where the balance between the up and down spins breaks. It should be noted that the spin unbalance occurs only
Table 1. Calculated Magnetic Moments Per Atom as a Function of Nanoplatelet Thickness

\begin{tabular}{lllll} 
thickness & $3.4 \mathrm{~nm}$ & $5.0 \mathrm{~nm}$ & $6.6 \mathrm{~nm}$ & $8.2 \mathrm{~nm}$ \\
magnetic & $<1.0 \times 10^{-3}$ & $1.0 \times 10^{-3}$ & $5.0 \times 10^{-3}$ & $<1.0 \times 10^{-3}$ \\
$\begin{array}{c}\text { moment } \\
\left(\mu_{\mathrm{B}} / \text { atom }\right)\end{array}$ & & & & \\
\hline
\end{tabular}

when the size along the $c$-axis is reduced. The development of an unbalanced spin distribution is not observed in a nanowire whose dimension is reduced along the $a$-and $b$-axes directions but not in $c$-axis direction. Considering the polarization at the $\mathrm{ZnO}\{0001\}$ top and bottom surfaces of the nanoplate, a very strong internal electric field can be expected within the thin body of the nanoplate, and such a high internal field is thought to be responsible for the unusual hybridization of $\mathrm{Zn}(3 \mathrm{~d})$ and $\mathrm{O}(2 \mathrm{p})$ bands with spin asymmetry. As the thickness increases, the magnitude of the internal field would decrease and its effect on the orbitals should reduce accordingly. For extremely thin nanoplates with thickness less than $5 \mathrm{~nm}$, there is a driving force to transform the wurtzite crystal structure to the graphitic structure ${ }^{20,21}$ that carries no dipoles in the $\mathrm{ZnO}$ layers. The transformation to graphitic structure was also confirmed in our calculation (see Supporting Information for more detail). Therefore, as the plates get extremely thin, the internal electric field reduces with the thickness of the plate, hence the reduced magnetic moment as shown in Figure 3a. The effect of internal electric field was further tested by varying the chemistry at the two $\{0001\}$ surfaces. With $\mathrm{Zn}$ and $\mathrm{OH}$ termination (as shown in Supporting Information), the amount of accumulated charge on the top and bottom surfaces change and the corresponding electric field strength changes. As expected, increased charge with $\mathrm{Zn}$ termination increases the magnetic moment and decreased charge with $\mathrm{OH}$ termination decreases the magnetic moment. On the other hand, calculation also shows that the presence of oxygen vacancies in bulk $\mathrm{ZnO}$ cannot produce any magnetism (see Supporting Information Figure S1).

Magnetic hysteresis loops of the plates were experimentally measured using a vibrating sample magnetometer equipped in a Quantum Design Physical Property Measurement System (PPMS-9T), confirming the presence of magnetic moments as suggested by the calculation results. First, the $\mathrm{GaN}$ thin film used for the growth of the $\mathrm{ZnO}$ nanoplates was measured to confirm that it did not produce the observed magnetism. Starting materials, HMTA and zinc nitrate, used for chemical 

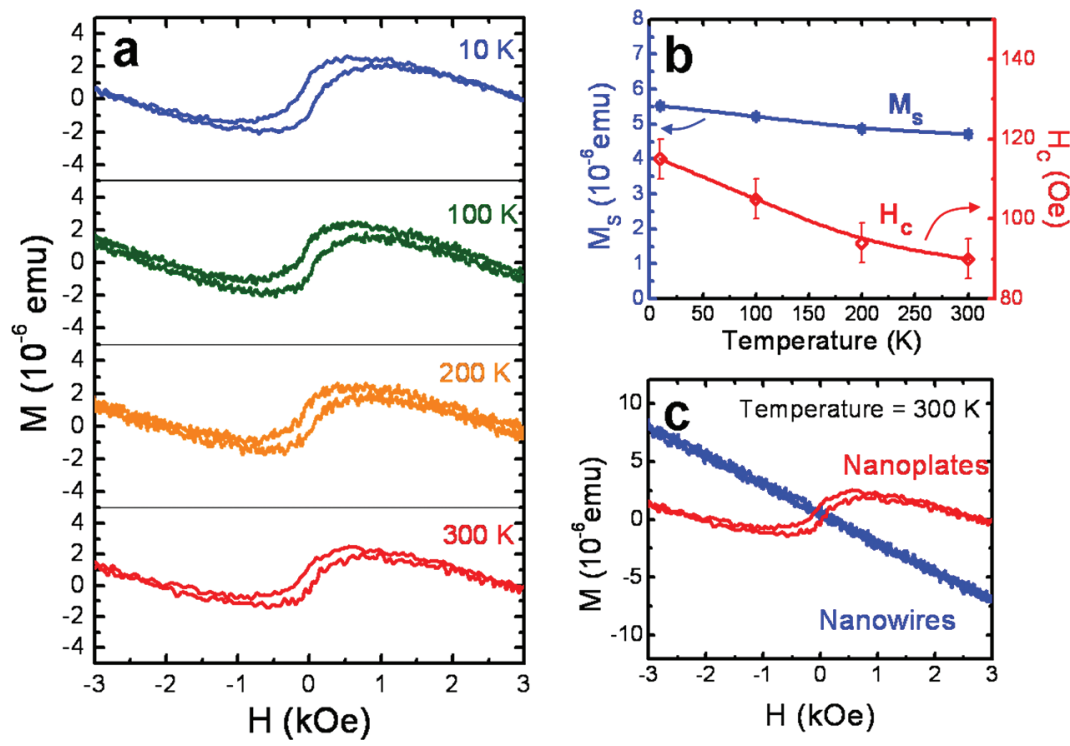

Figure 4. (a) Measured hysteresis loop of $\mathrm{ZnO}$ nanoplates at different temperatures ranging from 10 to $300 \mathrm{~K}$, clearly show the hysteresis loops. The corresponding $M_{\mathrm{S}}$ and coercivities $H_{\mathrm{C}}$ are plotted in (b). (c) The hysteresis loops measured from $\mathrm{ZnO}$ nanowires and nanoplates are compared, unambiguously showing that the ferromagnetism is present in nanoplates but not in nanowires. Magnetic measurement for nanowires in panel $\mathrm{c}$ gives only diamagnetic response with no hysteresis.
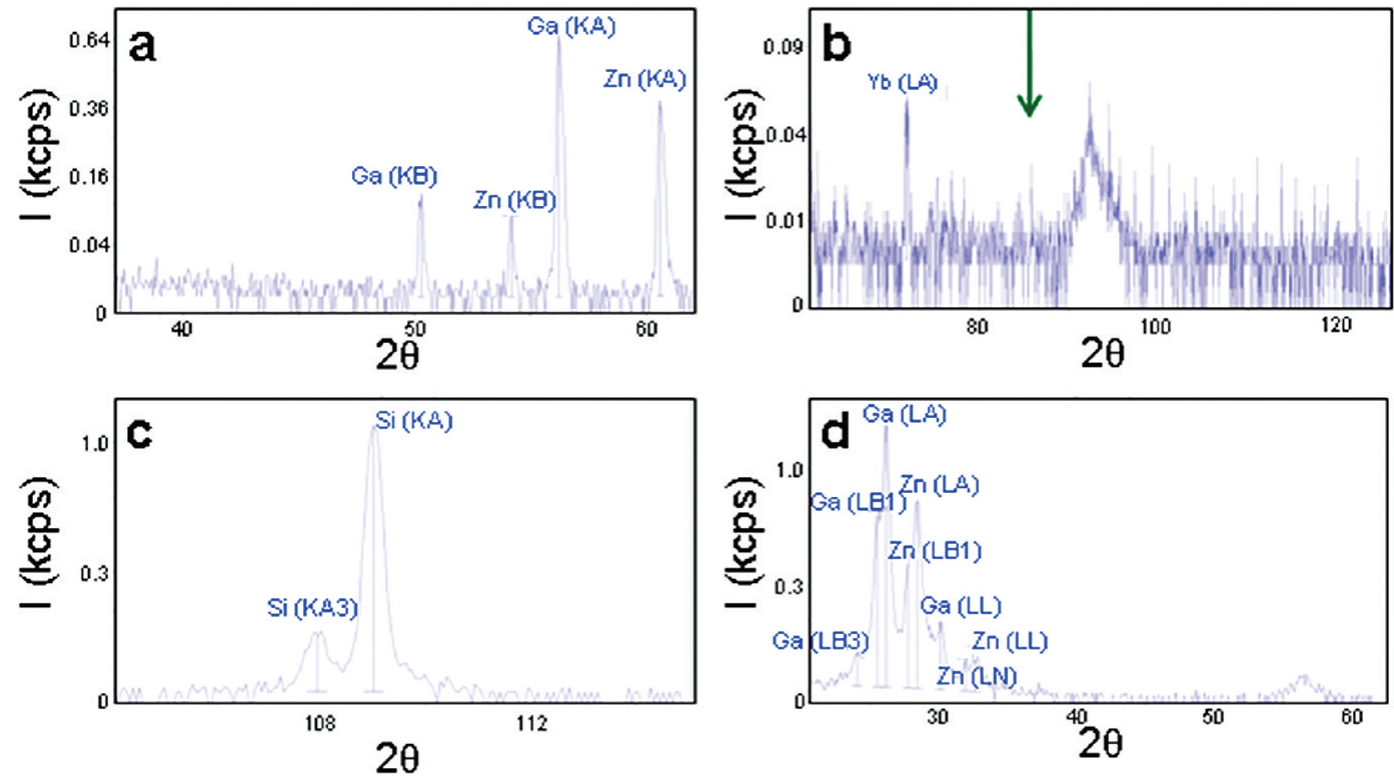

Figure 5. Measured peaks from the XRF scans run by the conditions in Supporting Information Table S1. Only the runs \#5, \#6, \#9, and \#11 showed measurable peaks that corresponds to $\mathrm{K} \alpha$ and $\mathrm{L} \alpha$ peaks of $\mathrm{Zn}, \mathrm{Ga}$, and $\mathrm{Si}$. No peaks corresponding to magnetic atoms (such as $\mathrm{Fe}, \mathrm{Ni}$, Co, or $\mathrm{Mn}$ ) could be detected.

synthesis of $\mathrm{ZnO}$ nanoplates were also measured to confirm that they are not contaminated with magnetic atoms (Supporting Information Figure S3).

As the synthesized specimen includes nanoplates with thickness variations, not all plates in the specimen are expected to be magnetic but a fraction of the plates with 5-8 nm thicknesses would be magnetic. Indeed, the measurable magnetic moment was measured at room temperature from the nanoplates grown on a $4 \mathrm{~mm} \times 4 \mathrm{~mm}$ substrate. Figure $4 \mathrm{a}$ shows the magnetic hysteresis loops of the nanoplates measured at various temperatures ranging from 10 to $300 \mathrm{~K}$. The temperature dependence of the saturated moment $\left(M_{\mathrm{S}}\right)$ and coercivity $\left(H_{\mathrm{C}}\right)$ is shown in Figure $4 \mathrm{~b}$.
It was recently pointed out that the experimental measurement of small nanomagnetic signals requires extreme care to exclude false signals from the sources related to the sample mounting and measurement procedures. ${ }^{22}$ Therefore, two measurements, before and after the specimen mounting (Figure S2 in Supporting Information), were taken and the background contributions including the duco cement used to mount the specimen on the sample holder was subtracted to confirm that the main contribution of the measurement came from the nanoplates. Because of the random distribution of the plates on the substrate, it was difficult to measure the exact weight amount of the nanoplates, but it was roughly estimated to be in the order of micrograms at most, based on the number density 
of the plates shown in the SEM micrographs. Therefore, the magnitude of the saturation magnetization is in the order of $10^{-1} \mathrm{emu} / \mathrm{g}$, which is much larger than the moments of $\mathrm{ZnO}$ based dilute magnetic semiconductors doped with magnetic atoms ${ }^{23}$ and is significantly larger than the measured moments originating from the broken bonds associated with the surface or atomic vacancies (typically in the order of less than $10^{-3}$ $\mathrm{emu} / \mathrm{g}$ ) in pure $\mathrm{ZnO}$ measured using nanoparticles ${ }^{24,25}$ or nanograined thin films. ${ }^{26}$ However, it is quite comparable to the calculated $M_{\mathrm{S}}$ of $0.069-0.34 \mathrm{emu} / \mathrm{g}$ obtained based on our first principles calculation discussed earlier, assuming the magnetic moment of $0.001-0.005 \mu_{\mathrm{B}}$ /atom depending on the thickness of the nanoplates as in Table 1. Alternatively, it was reported by Yi et al. ${ }^{27}$ that the presence of very small $\mathrm{Zn}$ clusters embedded in $\mathrm{ZnO}$ matrix may lead to the measurable magnetic moment in $\mathrm{ZnO}$. The magnitude of moment was comparable to the $M_{\mathrm{S}}$ of the nanoplates in the present work. We consider the possibility that a similar mechanism of the distorted band structure due to the electric field produced by charge accumulation at the interface between $\mathrm{Zn}$ cluster and $\mathrm{ZnO}$ matrix is responsible for the magnetic moment.

In the dilute magnetic semiconductor doped with magnetic atoms, the moment is associated with the dopant atoms. For the $\mathrm{ZnO}$ nanoplates, the moments are associated with all $\mathrm{Zn}$ and $\mathrm{O}$ atoms rather than impurities, hence the net moment is appreciable although the magnetization of individual atoms $\left(\sim 0.005 \mu_{\mathrm{B}}\right.$ /atom) is rather weak. In order to further ensure that the plates are free from magnetic contamination during the synthesis, inductively coupled plasma mass spectroscopy (ICP MS) and X-ray fluorescence (XRF) techniques were employed to confirm the chemical purity of the nanoplates synthesized. XRF scans (shown in Figure 5) show clear Ka and La peaks for $\mathrm{Zn}, \mathrm{Ga}$, and $\mathrm{Si}$ atoms in the sample. But no peaks corresponding to common magnetic atoms such as $\mathrm{Fe}, \mathrm{Co}$, and $\mathrm{Ni}$ could be measured. In Figure $5 \mathrm{~b}$, the position for a $\mathrm{Fe}$ peak is indicated with green line, showing the absence of measurable peak. The broad bump at $\sim 93^{\circ}$ was confirmed to be from the instrument, not from the specimen.

ICP mass spectroscopy provides a lower detection limit for the detection of trace elements. Therefore, the specimen was digested in a heated solution of nitric acid mixed with HF. Then the solution was analyzed with a Perkin-Elmer 9000 ICP MS. For Fe, Co, and Ni, the detection limits were 20, 0.4, and 1 ppm, respectively. The concentrations of those atoms in the specimens were all measured to be below the detection limits. Even if we assume that they could be present in the sample, at those low concentrations of magnetic ions the expected magnetic moment from those impurity atoms is 1 order of magnitude smaller than the measured magnetic moment, thus the measured hysteresis loop in Figure 4 cannot be explained by the presence of impurities. In order to further confirm, $\mathrm{ZnO}$ nanowires were also synthesized following the same chemical procedures $^{15}$ and the magnetic hysteresis loop of the nanowires was measured (see Figure 4c). In agreement with the calculated band structure shown in Figure 2a, the nanowires did not show any magnetic hysteresis behavior. This also assures that the chemical synthesis did not introduce magnetic impurities to the specimen. Therefore, all of the experimental results support the existence of a magnetic moment in the nanoplates but not in bulk or nanowires of $\mathrm{ZnO}$, and it is thought that the origin of the magnetic moment in the nanoplate is the modified electronic band structure due to the reduced dimension along the $c$-axis direction. This is consistent with the previous study by Garcia et al. ${ }^{28}$ in which modified surface electronic states of $\mathrm{ZnO}$ nanoparticle are responsible for a measurable magnetic moment in pure $\mathrm{ZnO}$,

In summary, it has been demonstrated that a magnetic moment can be induced into nominally nonmagnetic $\mathrm{ZnO}$ by reducing the dimension to a few nanometers along the $c$-axis direction. Size reduction of $\mathrm{ZnO}$ along the $a$ - or $b$ - axis direction results in the shape of a nanowire, which does not carry a magnetic moment. This experimental result is consistent with the first principles calculation, in which the symmetry between the up-spin and down-spin electrons in the electronic bands formed from the oxygen (2p) and zinc (3d) bands break in the $\mathrm{ZnO}$ nanoplates with thickness of 5 to $8 \mathrm{~nm}$. In addition to the well-known effect of overall size, the importance of anisotropic shape in the nanomaterial is established. This study presents an ideal example that unexpected physical properties can be created by controlling the size and shape of nanomaterials. Its discovery can have a broad impact to the fundamental studies and applications of many polar surface dominated crystals structures, such as $\mathrm{GaN}$ and $\mathrm{ZnS}$.

\section{ASSOCIATED CONTENT}

\section{S Supporting Information}

Additional information and figures. This material is available free of charge via the Internet at http://pubs.acs.org.

\section{AUTHOR INFORMATION}

\section{Corresponding Author}

*E-mail: (Z.L.W.) zhong.wang@mse.gatech.edu; (J.I.H.) jungil.hong@mse.gatech.edu; (S.S.J.) seungsoon.jang@mse.gatech. edu.

\section{ACKNOWLEDGMENTS}

The authors acknowledge the use of facility in the Center for Nanostructure Characterization (CNC) at Georgia Institute of Technology. The research was partially supported by BES DOE and NSF.

\section{REFERENCES}

(1) Geim, A. K.; Novoselov, K. S. Nat. Mater. 2007, 6, 183.

(2) Zhang, Y.; Tan, Y. W.; Stormer, H. L.; Kim, P. Nature 2005, 438, 201.

(3) Novoselov, K. S.; Geim, A. K.; Morozov, S. V.; Jiang, D.; Zhang, Y.; Dubonos, S. V.; Grigorieva, I. V.; Firsov, A. A. Science 2004, 306, 666.

(4) Lieber, C. M.; Wang, Z. L. MRS Bull. 2007, 32, 99.

(5) Wang, Z. L.; Kong, X. Y.; Ding, Y.; Gao, P.; Hughes, W. L.; Yang, R.; Zhang, Y. Adv. Funct. Mater. 2004, 14, 943.

(6) Tian, Z. R.; Voigt, J. A.; Liu, J.; McKenzie, B.; McDermott, M. J.; Rodrigues, M. A.; Konishi, H.; Xu, H. Nat. Mater. 2003, 2, 821.

(7) (a) Wang, Z. L. Ann. Rev. Phys. Chem. 2004, 55, 159. (b) Pan, Z. W.; Dai, Z. R.; Wang, Z. L. Science 2001, 291, 1947.

(8) Hong, J. I.; Bae, J.; Wang, Z. L.; Snyder, R. L. Nanotechnology 2009, 20, 085609.

(9) Xu, C. X.; Sun, X. W.; Dong, Z. L.; Yu, M .B. Appl. Phys. Lett. 2004, 85, 3878.

(10) Zhang, J.; Liu, H.; Wang, Z.; Ming, N.; Li, Z.; Biris, A. Adv. Funct. Mater. 2007, 17, 3897.

(11) Peng, Y.; Xu, A. W.; Deng, B.; Antonietti, M.; Colfen, H. J. Phys. Chem. B 2006, 110, 2988.

(12) Umar, A.; Hahn, Y. B. Nanotechnology 2006, 17, 2174.

(13) Ye, C.; Bando, Y.; Shen, G.; Golberg, D. J. Phys. Chem. B 2006, $110,15146$.

(14) Jing, Z.; Zhan, J. Adv. Mater. 2008, 20, 4547. 
(15) Xu, S.; Lao, C.; Weintraub, B.; Wang, Z. L. J. Mater. Res. 2008, 23, 2072.

(16) Hong, J. I.; Chang, Y.; Ding, Y.; Wang, Z. L.; Snyder, R. L. Thin Solid Films 2011, 519, 3608.

(17) (a) Kohn, W.; Sham, L. J. Phys. Rev. 1965, 140, A1133.

(b) Sham, L. J.; Kohn, W. Phys. Rev. 1966, 145, 561.

(18) Segall, M. D.; Lindan, P. J. D.; Probert, M. J.; Pickard, C. J.; Hasnip, P. J.; Clark, S. J.; Payne, M. C. J. Phys: Condens. Matter 2002, 14, 2717.

(19) (a) Yang, Y. H.; Chen, X. Y.; Feng, Y.; Yang, G. W. Nano Lett. 2007, 7, 3879. (b) Yousefi, R.; Kamaluddin, B. J. Alloys Compd. 2009, 479, L11.

(20) Freeman, C. L.; Claeyssens, F.; Allan, N L.; Harding, J. H. Phys. Rev. Lett. 2006, 96, 066102.

(21) Zhang, L.; Huang, H. Appl. Phys. Lett. 2007, 90, 023115.

(22) Garcia, M. A.; Fernandez Pinel, E.; de la Venta, J.; Quesada, A.; Bouzas, V.; Fernandez, J. F.; Romero, J. J.; Martin Gonzalez, M. S.; Costa-Kramer, J. L. J. Appl. Phys. 2009, 105, 013925.

(23) Pan, F.; Song, C.; Liu, X. J.; Yang, Y. C.; Zeng, F. Mater. Sci. Eng., $R$ 2008, 62, 1.

(24) Sundaresan, A.; Bhargavi, R.; Rangarajan, N.; Siddesh, U.; Rao, C. N. R. Phys. Rev. B 2006, 74, 161306(R).

(25) Madhu, C.; Sundaresan, A.; Rao, C. N. R. Phys. Rev. B 2008, 77, 201306(R).

(26) Straumal, B. B.; Mazilkin, A. A.; Protasova, S. G.; Myatiev, A. A.; Straumal, P. B.; Schutz, G.; van Aken, P. A.; Goering, E.; Baretzky, B. Phys. Rev. B 2009, 79, 205206.

(27) Yi, J. B.; Pan, H.; Lin, J. Y.; Ding, J.; Feng, Y. P.; Thongmee, S.; Liu, T.; Gong, H.; Wang, L. Adv. Mater. 2008, 20, 1170.

(28) Garcia, M. A; Merino, J. M.; Fernandez Pinel, E.; Quesada, A.; de la Venta, J.; Ruiz Gonzalez, M. L.; Castro, G. R.; Crespo, P.; Llopis, J.; Gonzalez-Calbet, J. M.; Hernando, A. Nano Lett. 2007, 7, 1489. 